\title{
Outcome predictors for patients with stage II/III gastric cancer who undergo gastrectomy and $S-1$ adjuvant chemotherapy
}

\author{
YOSHINORI FUJIWARA ${ }^{1,2}$, SHUICHI FUKUDA ${ }^{1}$, MASANORI TSUJIE ${ }^{1}$, KOTARO KITANI $^{1}$, \\ KEISUKE INOUE $^{1}$, TOMONORI HAYASHI ${ }^{3}$, HAJIME ISHIKAWA ${ }^{1}$, \\ MASAO YUKAWA $^{1}$ and MASATOSHI INOUE ${ }^{1}$
}

\author{
${ }^{1}$ Department of Digestive Surgery, Nara Hospital, Kindai University School of Medicine, Ikoma, Nara 630-0293; \\ ${ }^{2}$ Department of Digestive Surgery, Kawasaki Medical School, Kurashiki, Okayama 701-0192; ${ }^{3}$ Department of Pharmacy, \\ Nara Hospital, Kindai University School of Medicine, Ikoma, Nara 630-0293, Japan
}

Received May 25, 2016; Accepted February 13, 2017

DOI: $10.3892 / 01.2017 .6286$

\begin{abstract}
Predictors of survival in patients with stage II/III gastric cancer (GC) who received tegafur/gimeracil/oteracil (S-1) adjuvant chemotherapy (ACT) subsequent to gastrectomy were examined. Additionally, the association between dose intensity of S-1 and survival rate was investigated. A total of 62 patients with stage II/III gastric cancer were retrospectively evaluated, each of whom had received a curative D2 gastrectomy and S-1 ACT. The relative performance (RP; administered/planned S-1 doses x100\%), body mass index (BMI), prognostic nutritional index (PNI) and body weight (BW) were calculated, and the association of survival with these factors and other clinicopathological parameters was examined. The 1-year treatment continuation rate for S-1 was $94.2 \%$, excluding patients who experienced cancer recurrences during their ACT year. The initial S-1 reduction rate was $38.7 \%$. Patients with stage II/IIIA disease exhibited significantly improved 5-year overall survival rates compared with patients with stage IIIB GC, 81.6/73.7 vs. $33.8 \%(\mathrm{P}<0.01)$. No association between RP and survival was observed. BMI, BW and PNI were significantly decreased following surgery compared with preoperative states. In the univariate analysis, postoperative BW loss (BMI loss), pathological stage and $>7$ lymph node metastases were significantly associated with outcome $(\mathrm{P}<0.05)$; in the multivariate analysis, postoperative
\end{abstract}

Correspondence to: Dr Yoshinori Fujiwara, Department of Digestive Surgery, Kawasaki Medical School, 577 Matsushima, Kurashiki, Okayama 701-0192, Japan

E-mail: yyfujiwara@nifty.com

Abbreviations: ACT, adjuvant chemotherapy; BMI, body mass index; $\mathrm{CCr}$, creatinine clearance; PNI, prognostic nutritional index; $\mathrm{RP}$, relative performance; $\mathrm{BW}$, body weight

Key words: gastric cancer, adjuvant chemotherapy, tegafur/gimeracil/oteracil, survival predictors
BW loss $>10.6 \%$ and pathological stages were independent prognostic factors for survival. Continuing S-1 ACT for the full year exhibited a greater effect on survival compared with dosage. Early postoperative nutritional deterioration may decrease the survival rates in these patients.

\section{Introduction}

Gastric cancer (GC) is the fifth most common type of malignancy in the world, with 952,000 incident cases estimated to have occurred in 2012 (1). The standard treatment for stage II/III GC in Japan is gastrectomy with extended (D2) lymphadenectomy plus postoperativetegafur/gimeracil/oteracil (S-1) adjuvant chemotherapy (ACT) for 1 year, according to the Adjuvant Chemotherapy Trial of S-1 for Gastric Cancer (ACTS-GC) $(2,3)$. However, $30.6 \%$ of patients undergo cancer recurrences, $65.8 \%$ continue their S-1 treatments for a full year and $46.5 \%$ of patients are administered reduced doses of their recommended regimens $(2,3)$. The clinical parameters for continuing S-1 ACT in these patients has been the focus of several studies (4-6), as has the role of S-1 dose intensity in ACT subsequent to curative gastrectomy for advanced GC (7). However, the association between relative dose intensity or continuation of S-1 and patient survival has not been fully characterized.

The prognostic significance of a number of clinical parameters has been examined for patients with GC subsequent to radical surgery (8-15). Of these, the preoperative prognostic nutritional index (PNI) as a reflection of the immunological and nutritional condition of the patients has been associated with the outcomes of patients who undergo gastrectomy for a number of stages of GC $(10,11,16,17)$. Although the association between body mass index (BMI) and postoperative complications has been investigated (18-22), the association between BMI and long-term survival subsequent to curative gastrectomy remains unclear (23-28). The association between BMI and outcome in patients who received ACT for colon cancer has been demonstrated (29), but not the analogous association for patients with gastric cancer. 
In the present study, the dose-response effects of S-1 ACT on survival in patients who received gastrectomy for GC were clarified, and other clinical factors that affected their survival were analyzed, including BMI and PNI as nutritional parameters.

\section{Patients and methods}

Data collection involved a survey of original medical records and drug information databases of Nara Hospital, Kindai University School of Medicine (Ikoma, Japan). Patients with histologically confirmed primary gastric adenocarcinoma who had received curative gastrectomy with D2 lymphadenectomy and ACT with S-1 between January 2007 and December 2014 at Nara Hospital, Kindai University School of Medicine were enrolled and retrospectively evaluated. All patients exhibited adenocarcinoma histology, as demonstrated by endoscopic biopsies, with pathological stage II or III disease according to the Japanese Classification of Gastric Carcinoma (13th edition) (30); the TNM Classification of Malignant Tumors (7th edition) (31) was also used. Gastric adenocarcinomas can be divided into two major histological types, diffuse and intestinal type according to the Lauren classification (32). Patients received S-1 beginning between 4 and 8 weeks after surgery, typically at a standard dose of S-1 of $80 \mathrm{mg} / \mathrm{m}^{2} /$ day for 4 weeks, followed by 2 weeks of no chemotherapy. This 6-week cycle was repeated during the first year following surgery. A 3-week regimen of $80 \mathrm{mg} / \mathrm{m}^{2} \mathrm{~S}-1$ for 2 weeks, followed by a 1-week rest, was also permitted. Certain patients received $\mathrm{S}-1$ dose decreases, according to the criteria outlined in the ACTS-GC study (3), but decisions to decrease or cease S-1 ACT were entrusted to the patients and their physicians. Almost all patients in whom cancer recurrence was detected during their year of S-1 ACT were transferred to second-line chemotherapy, which included CPT-11, cisplatin and taxanes.

Toxicities were graded according to the National Cancer Institute Common Toxicity Criteria (version 3.0) (33). The present study was approved by the ethical review committee of Nara Hospital, Kindai University School of Medicine. Median follow up time was 44.76 months, ranging between 13.5-92 months. Written informed consent was obtained from all patients. The concept of the present study is available on the Nara Hospital website (http://www.kindainara. com/act/goannai.pdf).

Clinical and nutritional parameters. Clinical and pathological parameters were evaluated from medical records, and postoperative prognostic factors with S-1 adjuvant therapy were analyzed. The relative performance (RP) value was evaluated instead of the relative dose intensity. RP value was calculated as (administrated S-1 dose)/(planned S-1 administration dose) x100\%. The overall survival (OS) rates were compared between low- and high-RP groups. Creatinine clearance (CCr), was calculated using the formula developed by Cockroft and Gault (34). PNI was evaluated and calculated as $[10 \mathrm{x}$ serum albumin value $(\mathrm{g} / \mathrm{dl})]+$ (0.005x peripheral lymphocyte counts), using serum albumin $(\mathrm{mg} / \mathrm{dl})$ level and peripheral lymphocyte counts (counts $/ \mathrm{mm}^{3}$ )
Table I. Clinicopathological characteristics of patients in the present study $(\mathrm{n}=62)$.

Factor $\mathrm{n}$

Sex

Male

Female

Mean age, years

Tumor location in stomach

Upper

Middle

21

Lower

Pathological type ${ }^{a}$

Intestinal

Diffuse

Depth of tumor invasion $(\mathrm{pT})^{\mathrm{b}}$

T1

T2

11

T3

18

$\mathrm{T} 4 \mathrm{a}$

28

$\mathrm{T} 4 \mathrm{~b}$

Pathological nodal status ${ }^{\mathrm{c}}$

N0

N1

N2

No. of lymph node metastasis $(\mathrm{pN})^{\mathrm{c}}$

0 ( N0)

$1-2(\mathrm{~N} 1)$

3-6 (N2)

12

$\geq 7$ (N3)

Final pathological stage ${ }^{c}$

II

IIIA

IIB

Surgery type

Total gastrectomy

Distal gastrectomy

41

aLauren classification (32); ${ }^{\mathrm{b}} \mathrm{TNM}$ Classification of Malignant Tumours (7th edition) (31); ' Japanese Classification of Gastric Carcinoma (13th edition) (30). p, pathological; T, tumor; N, node.

assessed between 1 and 2 months after surgery (35), and BMI $\left(\mathrm{kg} / \mathrm{m}^{2}\right)$ was calculated as weight in $\mathrm{kg} /$ (height in $\left.\mathrm{m}\right)^{2}$. Body weight (BW), BMI and PNI were evaluated between 2 and 4 weeks before, and between 1 and 2 months after, surgery. Postoperative BW and BMI losses were calculated between 1 and 2 months after surgery as (postoperative value-preoperative value)/preoperative value x100\%). As BW loss and BMI loss were the same values, we evaluated only BW loss. The threshold values for postoperative BW loss (BMI loss), PNI, CCr and number of metastatic lymph nodes were decided by receiver operating characteristic (ROC) curves for OS. Other clinical and pathological prognostic 


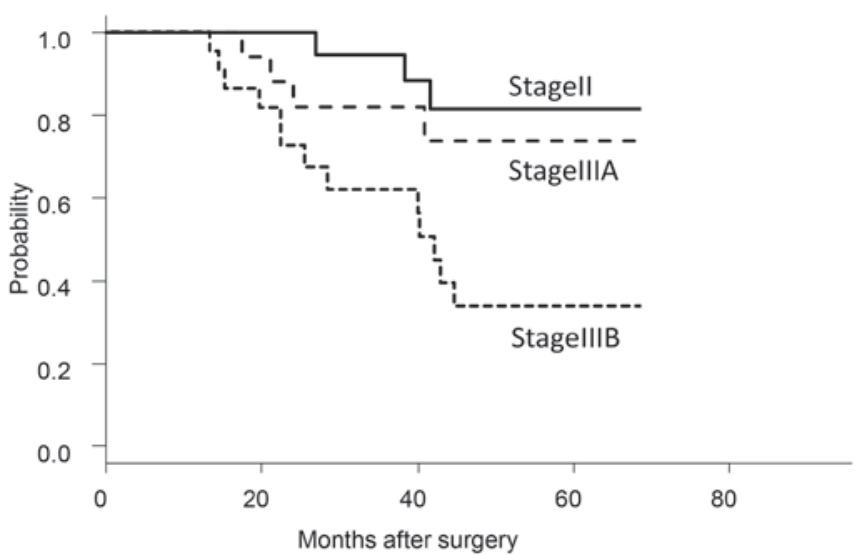

Figure 1. Patients with stage II-IIIA disease exhibited significantly improved 5 -year overall survival compared with patients with stage IIIB disease $(\mathrm{P}<0.01)$

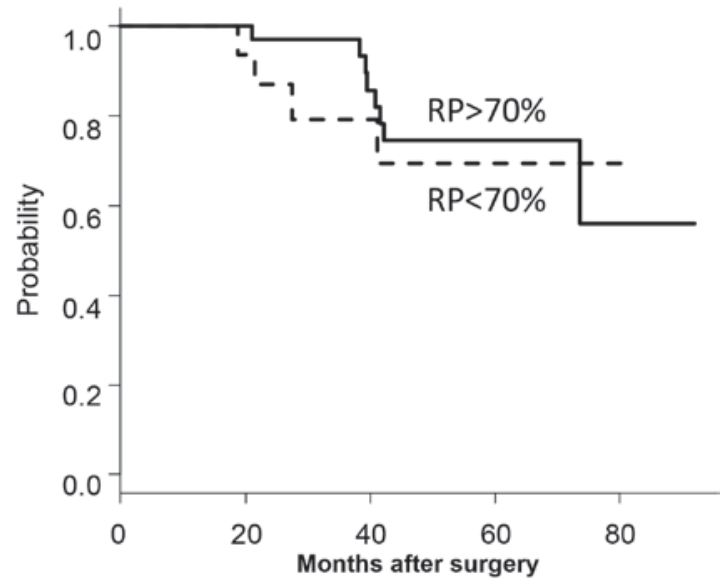

Figure 2. Overall survival by RP value for patients who underwent gastrectomies and S-1 adjuvant chemotherapy. Survival rates did not significantly differ by RP subsequent to exclusion of patients who experienced recurrences between 6 and 12 months after surgery. RP, relative performance.

factors included age, sex, type of gastrectomy and tumor stage (pStage) were also evaluated.

Statistical analysis. OS was defined as time between surgery and patient mortality or the last available information pertaining to vital status. Differences between cumulative survival rates of the patient groups were calculated using a log-rank test for comparison using Kaplan-Meier survival curves. $\mathrm{P}<0.05$ was considered to indicate a statistically significant difference. PNI, BMI, BW were expressed as the mean \pm standard deviation. The Wilcoxon signed rank test was used for comparison between preoperative and postoperative PNI, BMI and BW. Time-dependent survival ROC curves were used to evaluate the sensitivity and specificity of postoperative BW loss, CCr, PNI and number of metastatic lymph nodes for predicting the 3-year OS rate (36). The Youden index was used to determine optimal threshold values for postoperative BW loss, BMI loss, $\mathrm{CCr}$, number of metastatic lymph nodes and PNI. Patients were divided into two subgroups, above and below the threshold values, for each factor. Factors were also subjected to univariate and multivariate analyses, using Cox's proportional hazard model, against OS. Statistical analyses used JMP (version 11; SAS, Tokyo, Japan). Survival ROC was analyzed using R software (version 3.1.1; R Project for Statistical Computing, Vienna, Austria).

\section{Results}

A total of 62 patients were enrolled in the present study (Table I), all of whom received S-1 ACT as outpatients, at $60-120 \mathrm{mg} / \mathrm{day}$.

Survival. For the cohort of the present study, 3- and 5-year OS rates were 79.1 and $60.9 \%$, respectively, but varied according to disease stage (Fig. 1). The stage II and IIIA groups exhibited significantly improved 5-year OS rates (stage II, 81.6\%; stage IIIA, 73.7\%) compared with that of the stage IIIB group (33.8\%; $\mathrm{P}<0.01)$.

Recurrence. Of the 62 patients, 25 (40.3\%) experienced cancer recurrences. The sites of recurrence were peritoneal dissemination in 13 patients, liver in 5 patients, bone in 2 patients, lymph nodes in 4 patients, and lung in 1 patient.

ROC curves and threshold values. ROC survival curves were used to determine optimal threshold values for favorable OS in the factors under investigation. The threshold values were: PNI, 48 [area under curve (AUC), 0.637; true positive (TP), 0.96; false positive (FP), 0.57]; $\mathrm{CCr}, 70 \mathrm{ml} / \mathrm{min}$ (AUC, 0.541; TP, 0.72; FP, 0.485); BW loss, 10.6\% (AUC, 0.612; TP, 0.669; FP, 0.255) and number of metastatic lymph nodes, 7 (AUC, 0.733; TP, 0.645; FP, 0.183). The analyses of ROC curves used a 3-year endpoint and maximum Youden index.

As the ROC curves did not produce an optimal threshold value for BMI, it was set at $23 \mathrm{~kg} / \mathrm{m}^{2}$ according to the WHO Expert Consultation (37); the RP threshold value was set at $70 \%$ according to the ACTS-GC subset report (6). Patients were defined as those above or below each threshold and the two groups were compared.

$R P$ value and survival. Of the 62 patients, 24 were administered decreased S-1 doses within the first 3 cycles, at their or their physicians' decision, for an initial reduction rate of $38.7 \%$. A total of 3 patients elected to end their S-1 ACT regimens following 3 or 4 cycles (patient refusal); these patients did not exhibit cancer recurrences. The 1-year treatment continuation rate was $79.0 \%$, including patients who exhibited recurrences during S-1 ACT and were referred to second-line chemotherapies. Among the patients who did not exhibit recurrences, the 1-year treatment continuation rate was $94.2 \%$.

Of the 62 patients, 36 exhibited RP values of $>70 \%$, including 12 patients who possessed $100 \%$ RPs, and 26 patients exhibited RP values of $<70 \%$. A total of 16 patients in the $<70 \%$ RP group were lost to follow-up due to toxicity, and 10 for cancer recurrence leading to second-line chemotherapy between 6 and 12 months after surgery. The 5 -year OS rate of the $>70 \%$ RP group $(74.6 \%)$ was significantly increased compared with the $<70 \%$ RP group $(41.2 \%$; $\mathrm{P}<0.01)$. However, as the 10 patients who had stopped their S-1 ACT 
regimens due to recurrence between 6 and 12 months after surgery and were on second-line therapies were excluded, no significant difference between the 5-year OS rates for the $>70 \%$ RP group (74.7\%) and the $<70 \%$ RP group was identified (69.3\%; P=0.642; Fig. 2).

Nutritional parameters prior to and subsequent to surgery. Preoperative values were significantly decreased subsequent to surgery for BMI $\left(20.69 \pm 2.811\right.$ vs. $22.79 \pm 2.97 \mathrm{~kg} / \mathrm{m}^{2}$; $\mathrm{P}<0.001)$, BW (55.01 \pm 9.94 vs. $60.68 \pm 10.94 \mathrm{~kg} ; \mathrm{P}<0.001)$ and PNI (48.36 \pm 4.26 vs. $50.36 \pm 4.12$; P $<0.001$; Fig. 3).

Prognostic factors for OS. In the univariate analysis (Table II), BW loss between 1 and 2 months after surgery, pStage and number of lymph node metastases were of significant prognostic value $(\mathrm{P}<0.05)$. In multivariate analysis, BW loss between 1 and 2 months after surgery, and pStage were identified to be independent prognostic factors $(\mathrm{P}<0.05$, Table III).

\section{Discussion}

The present retrospective study was designed to evaluate the clinical prognostic factors of OS, including the nutritional parameters in gastrectomy patients who receive ACT with S-1 for GC in Nara Hospital. As described in the ASTS-GC reports, a 1-year regimen of S-1 ACT was effective for stage II and stage IIIA GC, but not stage IIIB disease, according to subset analysis $(2,3)$. In the present study, $94.2 \%$ of patients continued S-1 when the 10 patients who experienced cancer recurrence within the 1-year ACT period were excluded. However, the $38.7 \%$ of patients who decreased their S-1 doses within the first 3 cycles were included. The OS rate of the present study was similar to that of the ACTS-GC report, except for stage IIIB disease $(2,3)$.

It was revealed that, even if the S-1 dose was reduced, patients who continued to receive it for the recommended year exhibited a significant survival benefit. The lowest dose of S-1 administered in the present study was $60 \mathrm{mg} / \mathrm{day}$, for which a sufficient clinical effect has not been established; however, it should be established in the future. It is expected that more effective types of adjuvant therapy may be established in the near future for stage III disease. According to the ACTS-GC results [unpublished data, noted in (6)], improved survival was noted with 1-year continuation of S-1 ACT and RP values $>70 \%$. In the present study, the high and low RP groups did not significantly differ in OS when the patients with between 6 and 12-month postsurgical recurrences, i.e., those on second-line regimens, were excluded. Physicians in hospital settings may have decreased their patients' S-1 doses out of concern for toxic effects, which may have affected these numbers. Additionally, the present study involved relatively small numbers of patients, which may also have affected the results. However, no association between RP values and survival was observed in the present study. Additional examination may be required.

Pre- and postsurgical nutritional parameters were also evaluated. The deterioration in nutritional status subsequent to gastrectomy is induced by decreased food intake due to decreased capacity (38-40). Several nutritional parameters were markedly decreased subsequent to surgery in the present study. Body weight loss between 1 and 2 months after surgery
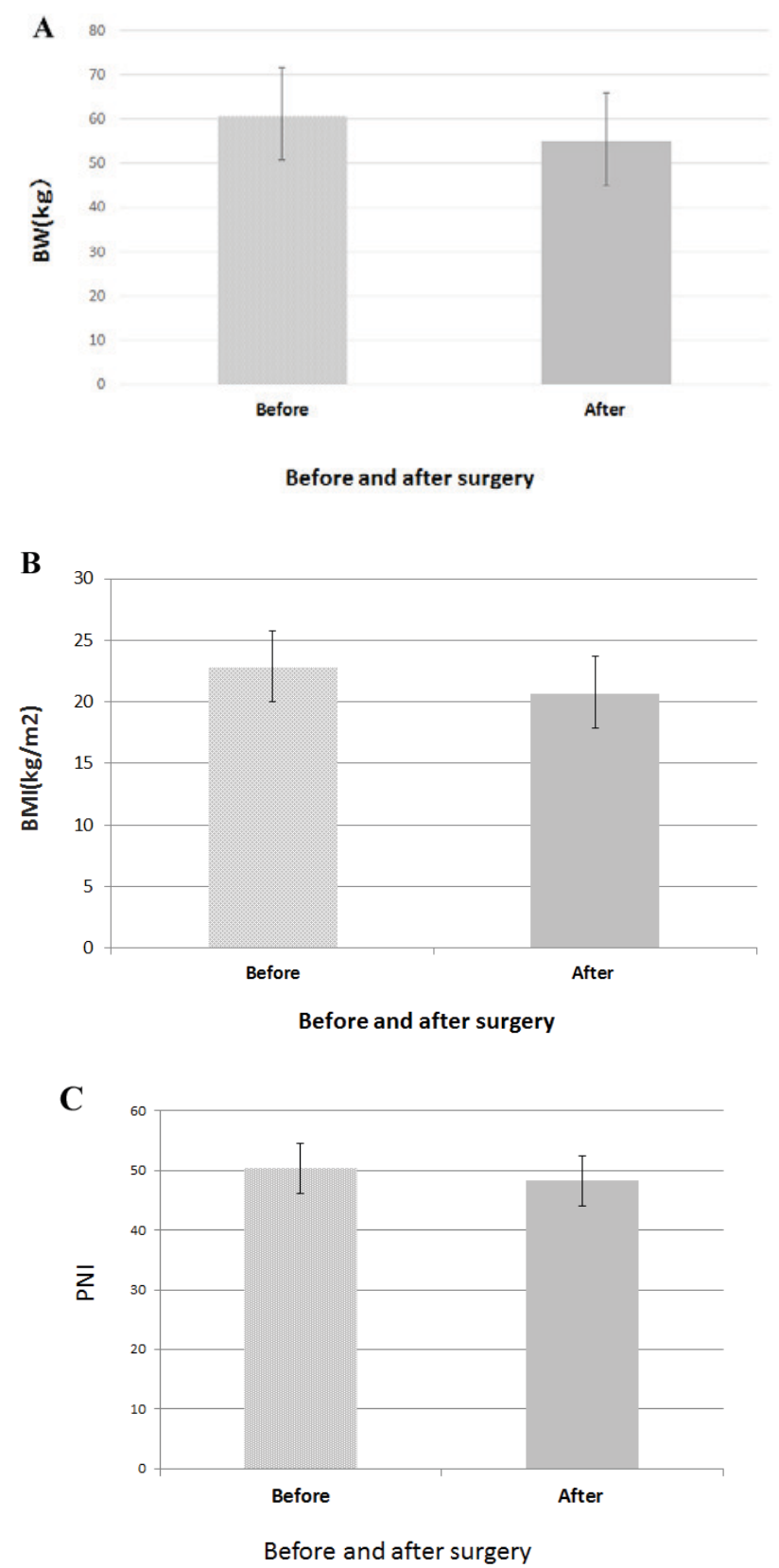

Figure 3. BW, BMI and PNI differences prior to and following surgery. (A) BW was significantly decreased at 1 and 2 months following gastrectomy compared with prior surgery $(\mathrm{P}<0.01)$. (B) BMI was significantly decreased at 1 and 2 months following gastrectomy compared with prior surgery $(\mathrm{P}<0.01)$. (C) PNI was significantly decreased at 1 and 2 months following gastrectomy compared with prior surgery $(\mathrm{P}<0.01)$. BW, body weight; $\mathrm{BMI}$, body mass index; PNI, prognostic nutritional index.

was a significant prognostic factor in the multivariate analysis for patients who received S-1 ACT. The prognosis of patients with BW loss $>10.6 \%$ between 1 and 2 months after surgery was poorer in the present study. Aoyama et al (5) demonstrated that BW loss of $>15 \%$ at 1 month after surgery was the most important risk factor for compliance of S-1 ACT. Additionally, BW loss at presentation is associated with poor chemotherapy compliance and poor prognosis in gastrointestinal malignancies (41). A previous study from Korea revealed that weight loss at the first month of palliative chemotherapy predicted unfavorable survival outcomes in patients with 
Table II. Univariate analysis of prognostic factors for overall survival in patients with gastric cancer who underwent neoadjuvant chemotherapy.

\begin{tabular}{|c|c|c|c|c|}
\hline Characteristic & $\mathrm{n}$ & HR & $95 \% \mathrm{CI}$ & P-value \\
\hline \multicolumn{5}{|l|}{ Age, years } \\
\hline$\geq 70$ & 18 & 1.013 & $0.392-2.616$ & 0.979 \\
\hline$<70$ & 44 & & & \\
\hline \multicolumn{5}{|l|}{ Sex } \\
\hline Male & 42 & 1.204 & $0.477-3.041$ & 0.696 \\
\hline Female & 20 & & & \\
\hline \multicolumn{5}{|c|}{ Type of gastrectomy } \\
\hline Distal & 41 & 0.596 & $0.242-1.472$ & 0.263 \\
\hline Total & 21 & & & \\
\hline \multicolumn{5}{|l|}{ pStage } \\
\hline II & 21 & 4.64 & $1.318-16.381$ & 0.017 \\
\hline III & 41 & & & \\
\hline \multicolumn{5}{|c|}{ Lymph node metastasis } \\
\hline$\geq 7$ & 27 & 2.97 & $1.219-7.236$ & 0.017 \\
\hline$<7$ & 35 & & & \\
\hline \multicolumn{5}{|c|}{ Creatinine clearance } \\
\hline$\geq 70$ & 36 & 0.441 & $0.168-1.162$ & 0.098 \\
\hline$<70$ & 26 & & & \\
\hline \multicolumn{5}{|c|}{ Postoperative BMI } \\
\hline$\geq 23$ & 14 & 0.582 & $0.171-1.980$ & 0.386 \\
\hline$<23$ & 38 & & & \\
\hline \multicolumn{5}{|c|}{ Postoperative PNI } \\
\hline$\geq 48$ & 37 & 0.522 & $0.215-1.265$ & 0.15 \\
\hline$<48$ & 21 & & & \\
\hline \multicolumn{5}{|c|}{ Preoperative BMI } \\
\hline$\geq 23$ & 27 & 1.179 & $0.496-2.802$ & 0.709 \\
\hline$<23$ & 35 & & & \\
\hline \multicolumn{5}{|c|}{ Preoperative PNI } \\
\hline$\geq 48$ & 44 & 0.716 & $0.262-1.956$ & 0.514 \\
\hline$<48$ & 18 & & & \\
\hline \multicolumn{5}{|c|}{ Body weight loss, $\%$} \\
\hline$\geq 10.6$ & 23 & 2.744 & $1.157-6.505$ & 0.022 \\
\hline \multicolumn{5}{|l|}{ (BMI loss) } \\
\hline$<10.6$ & 39 & & & \\
\hline \multicolumn{5}{|l|}{$\mathrm{RP}, \%$} \\
\hline$\geq 70$ & 36 & 0.752 & $0.226-2.506$ & 0.643 \\
\hline$<70$ & 16 & & & \\
\hline
\end{tabular}

BMI, body mass index; PNI, prognostic nutritional index; RP, relative performance, pStage, tumor stage; CI, confidence interval; HR, hazard ratio.

advanced GC (42). However, it was not possible to determine the potential association between $\mathrm{S}-1$ continuation and body weight loss between 1 and 2 months after surgery owing to the high S-1 continuation in the present study; however, BW loss between 1 and 2 months after surgery is hypothesized to affect the prognosis of patients who receive S-1 ACT subsequent to gastrectomy. To the best of our knowledge, the present study has provided the first evidence that BW loss between 1 and 2 months after surgery is associated with survival rate in patients with GC who received ACT. However, whether BW loss affects prognoses directly or indirectly, for example by decreasing the effectiveness of S-1, remains unclear. In any case, diminished nutritional status may worsen the prognosis of any patient with a serious illness, including gastric cancer. 
Table III. Multivariate analysis of clinicopathological factors compared with overall survival in patients with gastric cancer who underwent neoadjuvant chemotherapy.

\begin{tabular}{lccc}
\hline Factor & HR & 95\% CI & P-value \\
\hline Age & 0.980 & $0.927-1.036$ & 0.980 \\
pStage & 5.236 & $1.447-18.95$ & 0.012 \\
BW loss subsequent & 2.821 & $1.117-7.126$ & 0.028 \\
to surgery & & & \\
\hline
\end{tabular}

HR, hazard ratio; CI, confidence interval; pStage, tumor stage; BW, body weight.

The association between BMI and long-term outcome has been investigated with regard to certain malignancies $(43,44)$, including a number of controversial studies evaluating BMI and GC prognosis (23-28). In the present study, preoperative and postoperative BMI of patients with stage II/III GC were evaluated, although no association between BMI and survival rates was identified.

Preoperative PNI is hypothesized to be a prognostic marker for a number of malignancies, including long-term survival for patients with GC $(10,11,17,45)$. However, no association between either preoperative or postoperative PNI and survival in patients with GC who received S-1 ACT was demonstrated. As it was revealed that post-surgical BMI and BW loss were more reliable predictors of survival than PNI, the present study suggests that nutritional support subsequent to surgery improves survival rates in patients with stage II and III GC who expect to receive S-1 ACT.

In conclusion, 1-year continuation of S-1 ACT had an increased effect on survival compared with relative dose intensity, as demonstrated by RP value, for patients with stage II/III GC. Additionally, postoperative nutritional intervention may improve survival rates of these patients. A novel treatment strategy for stage III gastric cancer may be warranted.

\section{Acknowledgements}

The authors wish to thank the department's assistant, Mrs Akiko Kaku, and the pharmacologist, Mrs. Saori Nishiura, for their help with data collection from the patients.

\section{References}

1. WHO: GLOBOSCAN 2012: Estimated cancer incidence, mortality and prevalence worldwide in 2012 from http://globocan.iarc.fr/Pages/fact_sheets_cancer.aspx. Accessed February 6, 2014.

2. Sasako M, Sakuramoto S, Katai H, Kinoshita T, Furukawa H, Yamaguchi T, Nashimoto A, Fujii M, Nakajima T and Ohashi Y: Five-year outcomes of a randomized phase III trial comparing adjuvant chemotherapy with S-1 versus surgery alone in stage II or III gastric cancer. J Clin Oncol 29: 4387-4393, 2011.

3. Sakuramoto S, Sasako M, Yamaguchi T, Kinoshita T, Fujii M, Nashimoto A, Furukawa H, Nakajima T, Ohashi Y, Imamura $\mathrm{H}$, et al: Adjuvant chemotherapy for gastric cancer with S-1, an oral fluoropyrimidine. N Engl J Med 357: 1810-1820, 2007.
4. Aoyama T, Yoshikawa T, Hayashi T, Kuwabara H, Mikayama Y, Ogata T, Cho H and Tsuburaya A: Risk factors for 6-month continuation of S-1 adjuvant chemotherapy for gastric cancer. Gastric Cancer 16: 133-139, 2013.

5. Aoyama T, Yoshikawa T, Shirai J, Hayashi T, Yamada T, Tsuchida K, Hasegawa S, Cho H, Yukawa N, Oshima T, et al: Body weight loss after surgery is an independent risk factor for continuation of S-1 adjuvant chemotherapy for gastric cancer. Ann Surg Oncol 20: 2000-2006, 2013.

6. Kawabata R, Imamura H, Kishimoto T, Hachino Y, Yasui Y, Fujino M, Fujii C, Fukunaga M, Ohzato $\mathrm{H}$ and Furukawa $\mathrm{H}$ : Examination of factors influencing continuity of S-1 adjuvant chemotherapy for gastric cancer patients. Gan To Kagaku Ryoho 39: 1205-1208, 2012.

7. Kim SJ, Kim YJ, Kim JH, Park DJ, Kim HH, Lee JS and Lee KW: Safety, compliance, and predictive parameters for dosage modification in adjuvant S-1 chemotherapy for gastric cancer. Cancer Sci 104: 116-123, 2013.

8. Lien YC, Hsieh CC, Wu YC, Hsu HS, Hsu WH, Wang LS, Huang MH and Huang BS: Preoperative serum albumin level is a prognostic indicator for adenocarcinoma of the gastric cardia. J Gastrointest Surg 8: 1041-1048, 2004.

9. Liu H, Deng J, Zhang R, Hao X, Jiao X and Liang H: The RML of lymph node metastasis was superior to the LODDS for evaluating the prognosis of gastric cancer. Int J Surg 11: 419-424, 2013.

10. Migita K, Takayama T, Saeki K, Matsumoto S, Wakatsuki K, Enomoto K, Tanaka T, Ito M, Kurumatani N and Nakajima Y: The prognostic nutritional index predicts long-term outcomes of gastric cancer patients independent of tumor stage. Ann Surg Oncol 20: 2647-2654, 2013.

11. Sakurai K, Ohira M, Tamura T, Toyokawa T, Amano R, Kubo N, Tanaka H, Muguruma K, Yashiro M, Maeda K and Hirakawa K: Predictive potential of preoperative nutritional status in long-term outcome projections for patients with gastric cancer. Ann Surg Oncol 23: 525-533, 2016.

12. Ema A, Yamashita K, Sakuramoto S, Wang G, Mieno H, Nemoto M, Shibata T, Katada N, Kikuchi S and Watanabe M: Lymph node ratio is a critical prognostic predictor in gastric cancer treated with S-1 chemotherapy. Gastric Cancer 17: 67-75, 2014.

13. Pattison S, Mann GB, Crosthwaite G, Lade S, Mitchell C, Leong T, Busuttil RA and Boussioutas A: Predictors of outcome after surgery for gastric cancer in a Western cohort. ANZ J Surg 86: 469-474, 2016.

14. Maruyama K: The most important prognostic factors for gastric cancer patients: A study using univariate and multivariate analysis. Scandinavian J Gastroenterol 22: 63-68, 1987.

15. Kim JP,Lee JH, Kim SJ, Yu HJ and Yang HK: Clinicopathological characteristics and prognostic factors in 10783 patients with gastric cancer. Gastric Cancer 1: 125-133, 1998.

16. Nozoe T, Ninomiya M, Maeda T, Matsukuma A, Nakashima $H$ and Ezaki T: Prognostic nutritional index: A tool to predict the biological aggressiveness of gastric carcinoma. Surg Today 40: 440-443, 2010.

17. Watanabe $\mathrm{M}$, Iwatsuki $\mathrm{M}$, Iwagami $\mathrm{S}$, Ishimoto $\mathrm{T}$, Baba $\mathrm{Y}$ and Baba H: Prognostic nutritional index predicts outcomes of gastrectomy in the elderly. World J Surg 36: 1632-1639, 2012.

18. Kodera Y, Sasako M, Yamamoto S, Sano T, Nashimoto A and Kurita A; Gastric Cancer Surgery Study Group of Japan Clinical Oncology Group: Identification of risk factors for the development of complications following extended and superextended lymphadenectomies for gastric cancer. Br J Surg 92: 1103-1109, 2005.

19. Gretschel S, Christoph F, Bembenek A, Estevez-Schwarz L, Schneider U and Schlag PM: Body mass index does not affect systematic D2 lymph node dissection and postoperative morbidity in gastric cancer patients. Ann Surg Oncol 10: 363-368, 2003.

20. Barry JD, Blackshaw GR, Edwards P, Lewis WG, Murphy P, Hodzovic I, Thompson IW and Allison MC: Western body mass indices need not compromise outcomes after modified D2 gastrectomy for carcinoma. Gastric Cancer 6: 80-85, 2003.

21. Inagawa $\mathrm{S}$, Adachi $\mathrm{S}$, Oda $\mathrm{T}$, Kawamoto T, Koike $\mathrm{N}$ and Fukao K: Effect of fat volume on postoperative complications and survival rate after D2 dissection for gastric cancer. Gastric Cancer 3: 141-144, 2000.

22. Lee JH, Paik YH, Lee JS, Ryu KW, Kim CG, Park SR, Kim YW, Kook MC, Nam BH and Bae JM: Abdominal shape of gastric cancer patients influences short-term surgical outcomes. Ann Surg Oncol 14: 1288-1294, 2007. 
23. Kodera Y, Ito S, Yamamura Y, Mochizuki Y, Fujiwara M, Hibi K, Ito K, Akiyama S and Nakao A: Obesity and outcome of distal gastrectomy with D2 lymphadenectomy for carcinoma. Hepatogastroenterology 51: 1225-1228, 2004.

24. Dhar DK, Kubota H, Tachibana M, Kotoh T, Tabara H, Masunaga R, Kohno $\mathrm{H}$ and Nagasue N: Body mass index determines the success of lymph node dissection and predicts the outcome of gastric carcinoma patients. Oncology 59: 18-23, 2000.

25. Murphy PM, Blackshaw GR, Paris HJ, Edwards P, Barry JD and Lewis WG: Prospective evaluation of nutritional status related to body mass indices and outcomes after modified D2 gastrectomy for carcinoma. Clin Nutr 23: 477-483, 2004.

26. Tokunaga M, Hiki N, Fukunaga T, Ohyama S, Yamaguchi T and Nakajima T: Better 5-year survival rate following curative gastrectomy in overweight patients. Ann Surg Oncol 16 3245-3251, 2009.

27. Nozoe T, Kohno M, Iguchi T, Mori E, Maeda T, Matsukuma A and Ezaki T: Analysis of the impact of the body mass index in patients with gastric carcinoma. Surg Today 42: 945-949, 2012

28. Moriwaki Y, Kunisaki C, Kobayashi S, Harada H, Imai S and Kasaoka C: Does body mass index (BMI) influence morbidity and long-term survival in gastric cancer patients after gastrectomy? Hepatogastroenterology 50: 284-288, 2003.

29. Dignam JJ, Polite BN, Yothers G, Raich P, Colangelo L, O'Connell MJ and Wolmark N: Body mass index and outcomes in patients who receive adjuvant chemotherapy for colon cancer. J Natl Cancer Inst 98: 1647-1654, 2006.

30. Japanese Gastric Cancer Association. Japanese classification of gastric carcinoma: 13th edition Kanehara, Tokyo, Japan, 1999.

31. Sobin LH, Gospodarowics MK and Wittekind CH: TNM classification of malignant tumours, 7th edition. John Wiley \& Sons, New York, 2009.

32. Lauren P: The two histologic main types of gastric carcinoma: Diffuse and so-called intestinal-type carcinoma. An attempt at a Histo-Clinical classification. Acta Parhol Microbid Scan 64 31-49, 1965.

33. Common terminology criteria for adverse events v3.0 (CTCAE) form http://ctep.cancer.gov/protocolDevelopment/electronic_ applications/docs/ctcaev3.pdf. Journal August 9, 2006.

34. Cockcroft DW and Gault MH: Prediction of creatinine clearance from serum creatinine. Nephron 16: 31-41, 1976.
35. Onodera T and Goseki N and Kosaki G: Prognostic nutritional index in gastrointestinal surgery of malnourished cancer patients. Nihon Geka Gakkai Zasshi 85: 1001-1005, 1984 (In Japanese).

36. survivalROC: Time-dependent ROC curve estimation from censored survival data. Journal Accessed May 6, 2016 Available fromhttps://cran.r-project.org/web/packages/survivalROC/index. html.

37. WHO Expert Consultation: Appropriate body-mass index for Asian populations and its implications for policy and intervention strategies. Lancet 363: 157-163, 2004.

38. Kiyama T, Mizutani T, Okuda T, Fujita I, Tokunaga A, Tajiri T and Barbul A: Postoperative changes in body composition after gastrectomy. J Gastrointest Surg 9: 313-319, 2005.

39. Hirao M, Takiguchi S, Imamura H, Yamamoto K, Kurokawa Y, Fujita J, Kobayashi K, Kimura Y, Mori M and Doki Y; Osaka University Clinical Research Group for Gastroenterological Study: Comparison of Billroth I and Roux-en-Y reconstruction after distal gastrectomy for gastric cancer: One-year postoperative effects assessed by a multi-institutional RCT. Ann Surg Oncol 20: 1591-1597, 2013.

40. Hiki N: Body weight loss in cancer patients: Mechanism of body weight loss by treatment. The Japanese Journal of Clinical Nutrition. 120: 848-851, 2012 (In Japanese).

41. Andreyev HJ, Norman AR, Oates J and Cunningham D: Why do patients with weight loss have a worse outcome when undergoing chemotherapy for gastrointestinal malignancies? Eur J Cancer 34: 503-509, 1998.

42. Ock CY, Oh DY, Lee J, Kim TY, Lee KH, Han SW, Im SA, Kim TY and Bang YJ: Weight loss at the first month of palliative chemotherapy predicts survival outcomes in patients with advanced gastric cancer. Gastric Cancer 19: 597-606, 2016.

43. Sinicrope FA, Foster NR, Sargent DJ, O'Connell MJ and Rankin C: Obesity is an independent prognostic variable in colon cancer survivors. Clin Cancer Res 16: 1884-1893, 2010.

44. Dawood S, Broglio K, Gonzalez-Angulo AM, Kau SW, Islam R, Hortobagyi GN and Cristofanilli M: Prognostic value of body mass index in locally advanced breast cancer. Clin Cancer Res 14: 1718-1725, 2008.

45. Nozoe T, Kohno M, Iguchi T, Mori E, Maeda T, Matsukuma A and Ezaki T: The prognostic nutritional index can be a prognostic indicator in colorectal carcinoma. Surg Today 42: 532-535, 2012. 